\title{
Quantitative EEG evaluation in patients with acute encephalopathy
}

\author{
Análise quantitativa do EEG em pacientes com encefalopatias agudas \\ Aline Souza Marques da Silva Braga, Bruno Della Ripa Rodrigues Assis, Jamil Thiago Rosa Ribeiro, \\ Patrícia Maria Sales Polla, Breno José Hulle Pereira, Ronaldo Guimarães Fonseca, Luiz Eduardo Betting
}

\begin{abstract}
Objective: To investigate the use of quantitative EEG (qEEG) in patients with acute encephalopathies (AEs) and EEG background abnormalities. Method: Patients were divided into favorable outcome (group A, 43 patients) and an unfavorable outcome (group B, 5 patients). EEGLAB software was used for the qEEG analysis. A graphic of the spectral power from all channels was generated for each participant. Statistical comparisons between the groups were performed. Results: In group A, spectral analysis revealed spectral peaks (theta and alpha frequency bands) in 84\% (38/45) of the patients. In group B, a spectral peak in the delta frequency range was detected in one patient. The remainder of the patients in both groups did not present spectral peaks. Statistical analysis showed lower frequencies recorded from the posterior electrodes in group B patients. Conclusion: qEEG may be useful in the evaluations of patients with AEs by assisting with the prognostic determination.
\end{abstract}

Keywords: encephalopaties, coma, EEG, neurophysiology.

\section{RESUMO}

Objetivos: Investigar o uso do EEG quantitativo (qEEG) em pacientes com encefalopatias agudas (EAs) e anormalidades da atividade de base no EEG. Método: Os pacientes foram divididos em prognóstico favorável (grupo A, 43 pacientes) e desfavorável (grupo B, 5 pacientes). 0 programa EEGLAB foi utilizado para a análise do qEEG. Um gráfico da potência espectral de todos os canais foi gerado para cada participante. Os dois grupos foram comparados estatisticamente. Resultados: No grupo A, a análise espectral revelou picos (frequências teta e alfa) em $84 \%$ (38/45) dos pacientes. No grupo B, um pico espectral na frequência delta foi detectado em um paciente. Os pacientes remanescentes dos dois grupos não apresentaram picos espectrais. A análise estatística mostrou menores frequências registradas nos eletrodos posteriores dos pacientes do grupo B. Conclusão: O qEEG pode ser útil na avaliação de pacientes com EAs auxiliando na determinação do prognóstico.

Palavras-chave: encefalopatias, coma, EEG, neurofisiologia.

Acute encephalopathy $(\mathrm{AE})$ is a generic term that is often used to describe a diffuse cerebral abnormality. There are multiple etiologies for AEs, including metabolic, iatrogenic and toxic, infectious, primary neurologic, surgery-related, and others ${ }^{1}$. The main clinical feature of AEs is an altered state of consciousness that may range from minimally reduced wakefulness or awareness to coma ${ }^{1,2}$. Patients with this condition are frequently present in hospitals of any size, and their management requires a precise etiological diagnosis.

Although less attention has been paid to the EEG features characteristic of AEs compared to others such as epileptiform activity, seizures and status epilepticus, the usefulness of EEG in the evaluation of abnormal states of consciousness has been demonstrated ${ }^{3}$. It is well established that the amplitude and frequency of brain waves, or oscillations, that result from neural activity, and the particular patterns of the EEG, are closely correlated with the level of consciousness and with a deepening of coma $^{2,4}$. EEG is most helpful in the diagnosis of non-convulsive status epilepticus (NCSE) and in conditions such as hepatic encephalopathy or other metabolic disorders that can manifest with certain patterns such as triphasic waves ${ }^{5,6}$. EEG is also useful in prognostic evaluation. For example, EEG patterns such as burst-suppression have been associated with the highest mortality rates?

One common finding in patients with AEs is a diffuse slowing of oscillatory brain activity, an abnormality that is always present in metabolic coma ${ }^{4}$. When this pattern occurs in patients with subtle abnormalities of conscience, the

Departamento de Neurologia, Psiquiatria e Psicologia, Faculdade de Medicina de Botucatu, Universidade Estadual Paulista (UNESP), Sao Paulo SP, Brasil. Correspondence: Luiz Eduardo Betting; Departamento de Neurologia / Psiquiatria e Psicologia / Faculdade de Medicina de Botucatu - UNESP; $18618-970$ Botucatu SP - Brasil; E-mail: betting@fmb.unesp.br

Conflict of interest: There is no conflict of interest to declare.

Support: This study was supported by Fundação de Amparo à Pesquisa do Estado de São Paulo (FAPESP).

Received 14 January 2013; Received in final form 24 June 2013; Accepted 01 July 2013. 
differentiation between diffuse slowing and somnolence may be difficult ${ }^{8}$. Diffuse slowing may be both qualitatively and quantitatively assessed, and has a high degree of correlation with prognosis, particularly when the patient's etiology includes cardiorespiratory arrest ${ }^{4,9}$.

The progressive use of EEG in the emergency room and in the intensive care unit (sometimes with continuous monitoring) has expanded rapidly in the past several years. Thus, there is a need to increase our understanding of correlations that may exist between the EEG abnormalities observed in AEs and the clinical and laboratory features of these pathologies, including the prognosis of the patient ${ }^{3}$. The objective of the present work is to investigate qualitative and quantitative EEG patterns in patients with AEs and background abnormalities independently of disease etiology.

\section{METHOD}

The current study reviewed all the urgent EEGs performed in a one year interval as well as the medical records of the patients involved. Exams were performed in the EEG laboratory using a 22 channel recorder (Meditron; São Paulo, Brazil) following the 10-20 international system of electrode placement, including photic-stimulation and 20 to $30 \mathrm{mi}-$ nutes extent. The sampling rate used was $200 \mathrm{~Hz}$, and all electrode impedance was kept below $10 \mathrm{~K} \Omega$. This study was approved by the local Ethics Committee. The criteria for patient inclusion were: a diagnosis of AE defined as any abnormality of conscience continuing until the time of EEG evaluation, EEG and medical records that were available for review, EEGs showing background abnormalities characterized mainly by diffuse slow theta and delta waves, and no use of sedative medications for at least 24 hours prior to the EEG. Patients with clear symptoms of somnolence and/or with physiologic sleep patterns, who had been evaluated for brain death, who had a coma diagnosis or more specific EEG abnormalities such as triphasic waves, periodic discharges, NCSE, coma patterns (alpha, theta and spindle), burst suppression, or others were excluded from this investigation.

Patients were clinically classified into three main categories according to previously described definitions: clouding of consciousness (minimally reduced wakefulness or awareness), obtundation (mild to moderate reduction in alertness), and stupor (able to be aroused only with vigorous and continuous stimulation $)^{2}$.

Patients were divided into two groups: Group A patients had a favorable outcome (hospital discharge), and Group B patients had an unfavorable outcome (death).

\section{Qualitative EEG analysis}

All records included in this study were visually analyzed by one rater who was unaware of the clinical data. Exams were classified into 5 grades: 0- normal; 1- normal - limit (unstable or suppressed alpha rhythm frequently replaced by a high prevalence of diffuse beta rhythm); 2- mild signs of encephalopathy (low frequency alpha rhythm disturbed by random waves in the theta range over both hemispheres); 3- distinctive features of encephalopathy (background activity in the theta range, diffused over both hemispheres, and the random appearance of high amplitude waves in the delta range); 4- signs of severe encephalopathy (severe disorganization of EEG activity with no normal elements; having diffuse asynchronous theta and delta waves over both hemispheres $)^{10}$.

\section{Quantitative EEG analysis}

To perform the qEEG analysis, the EEGLAB version 9 (http://www.sccn.ucsd.edu/eeglab) software running under Matlab R2010a (MathWorks; Natick, USA) was used ${ }^{11}$. Twenty electrodes were used for this evaluation (Fp1, Fp2, F7, F3, Fz, F4, F8, T3, C3, Cz, C4, T4, T5, P3, Pz, P4, T6, O1, Oz, and $\mathrm{O} 2$ ) with linked $\mathrm{A} 1$ and $\mathrm{A} 2$ as references. All exams were subjected to the same pre-processing steps. First, exams were converted to European Data Format (EDF) and imported into the software. Then, the exams were filtered using a $0.5 \mathrm{~Hz}$ (low) and $50 \mathrm{~Hz}$ (high) cutoff frequency. Each exam was then submitted to artifact rejection by visual analysis. The localization of electrode coordinates were determined and corrected if necessary. Exams were then decomposed using independent component analysis ${ }^{11}$. Independent components were used for artifact rejection and for statistical analysis. Finally, for each participant, channel spectra and associated topographical maps were plotted using the fast Fourier transform. This generated data represents the whole record (20 to 30 minutes) within the frequencies of 2 and $24 \mathrm{~Hz}$, with emphasis in the alpha range (8-12 Hz).

To perform the quantitative evaluation, a statistical analysis was conducted that compared the EEG power spectrum and scalp maps of patients in Group A versus Group B using EEGLAB software. A cluster approach was also conducted to reinforce the statistical analysis. Independent components from each dataset were identified and grouped based on the mean of the scalp maps and power spectrum using principal component analysis ${ }^{12}$. Finally, a k-means algorithm was used to generate the clusters ${ }^{12,13}$. Comparisons were performed using a permutation test with 5000 permutations and the level of significance was set at $\mathrm{p}<0.05$.

\section{Statistical analysis}

Clinical differences including age, number of comorbidities, etiology, and clinical features were compared between Groups A and B. Qualitative EEG findings were also compared between the two groups. In the statistical analysis, we used Fisher's exact test to evaluate proportions, and the Mann-Whitney non-parametric test for means comparisons. 


\section{RESULTS}

\section{Clinical features}

Group A consisted of 43 patients (20 women, mean age $57 \pm 23$ years, range $18-93$ ). The mean number of comorbidities was $2 \pm 1$ (range 0 - 7). Ten patients in Group A were diagnosed with structural encephalopathy secondary to cerebrovascular disease, eight patients had metabolic disorders, six patients had systemic infectious diseases, six patients had epilepsy decompensated by infectious diseases, five patients had infections of the central nervous system, two patients had eclampsia, two patients had autoimmune encephalitis, two patients presented with traumatic brain injury, and two patients had hypoxia secondary to cardiac abnormalities. Group B consisted of five patients (two women, mean age $63 \pm 13$ years, range $48-83$ ). The mean number of comorbidities was $4 \pm 1$ (range $3-5$ ). All patients presented with systemic infectious diseases. Statistical analysis did not show significant differences between the two groups in terms of age ( $p=0.7, U=95.5$ ). The number of comorbidities was higher in Group B patients ( $\mathrm{p}=0.02, \mathrm{U}=42$ ).

Analysis of clinical features showed that 27 patients presented with clouding of consciousness, 10 with obtundation, and six with stupor in Group A. In Group B, two patients presented with clouding of consciousness and three with stupor. Group B contained a higher proportion of patients with a more severe clinical picture characterized by stupor $(p=0.04)$.

Since all patients in group B had systemic infectious diseases, a comparison with six patients (two women, mean age $64 \pm 28$ years, range 18 - 93) in group A with the same diagnosis was conducted. The mean number of comorbidities was $1 \pm 0.8$ (range $1-3$ ). Two patients presented with a clouding of consciousness, two with obtundation, and two with stupor. Compared with group B, statistical analysis showed difference only in the number of comorbidities $(\mathrm{p}=0.01, \mathrm{U}=1)$.

\section{Qualitative EEG analysis}

In Group A, seven (16\%) exams were rated as Grade 1 (unstable or suppressed alpha rhythm frequently replaced by a high prevalence of diffuse beta rhythm), 14 (32\%) as Grade 2 (low frequency alpha rhythm disturbed by random waves in the theta range over both hemispheres), 15 (35\%) as Grade 3 (background activity in the theta range, diffused over both hemispheres, and the random appearance of high amplitude waves in the delta range) and $7(16 \%)$ as Grade 4 (severe disorganization of EEG activity with no normal elements; having diffuse asynchronous theta and delta waves over both hemispheres $)^{10}$. Group B had a higher proportion of exams that were rated as Grade 4 (5 EEGs, $100 \%$, p $<0.001$ ). Figure 1 shows examples of the four grades of EEG exams.

In the patients of group A with systemic infectious diseases two EEGs were rated as Grade 2, two as Grade 3 and two as Grade 4. No statistical difference was observed when compared to group B ( $\mathrm{p}=0.5)$.

\section{Quantitative EEG analysis}

The visual evaluation of the spectral EEG data showed that, in Group A, 84\% (36/43) of the patients had anterior-posterior differentiation signs and spectral peaks in the theta and alpha bands (Figure 2A). In Group B, a spectral peak in the delta band was detected in one patient, and the remainder of the patients in both groups did not present spectral peaks (Figure 2B).

Statistical analysis confirmed the observations described above. Comparisons between the two groups revealed that the main differences consisted of lower frequencies in the posterior electrodes and reduced power spectrum in the Group B patients, compared to Group A (Figure 3A and B superior row, Figure 4A).

Evaluating the patients of group A with systemic infectious diseases, spectral peaks in theta and alpha bands were present

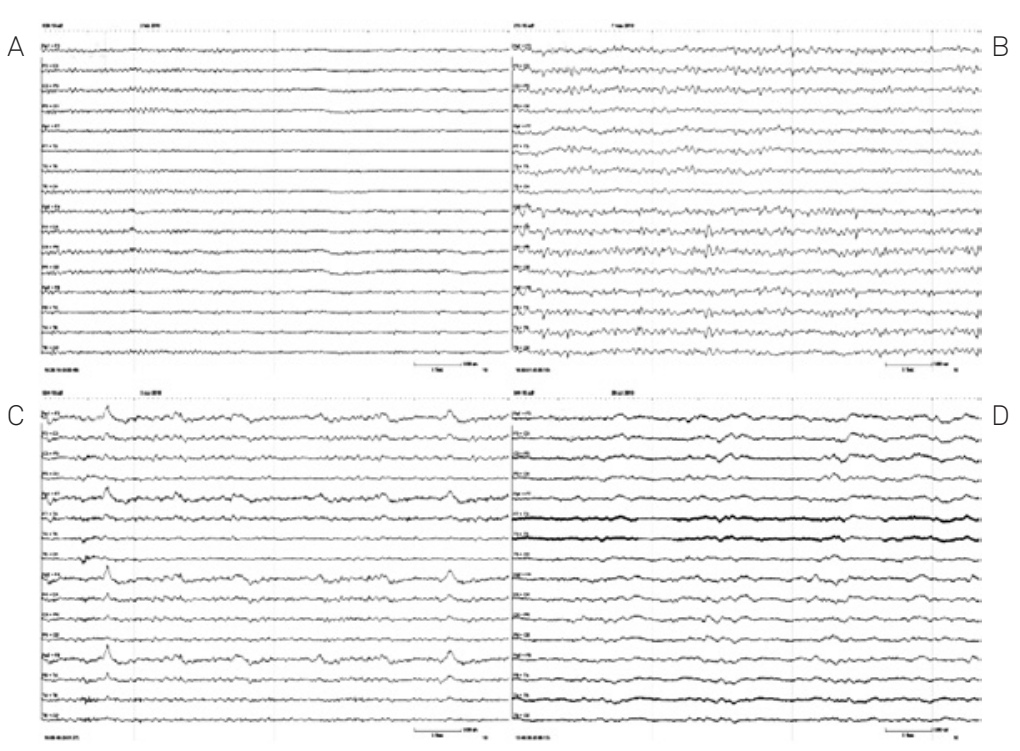

Figure 1. Examples of the EEG classifications in patients with acute encephalopathy ${ }^{10}$. A) Grade 1; B) Grade 2; C) Grade 3; D) Grade 4. 

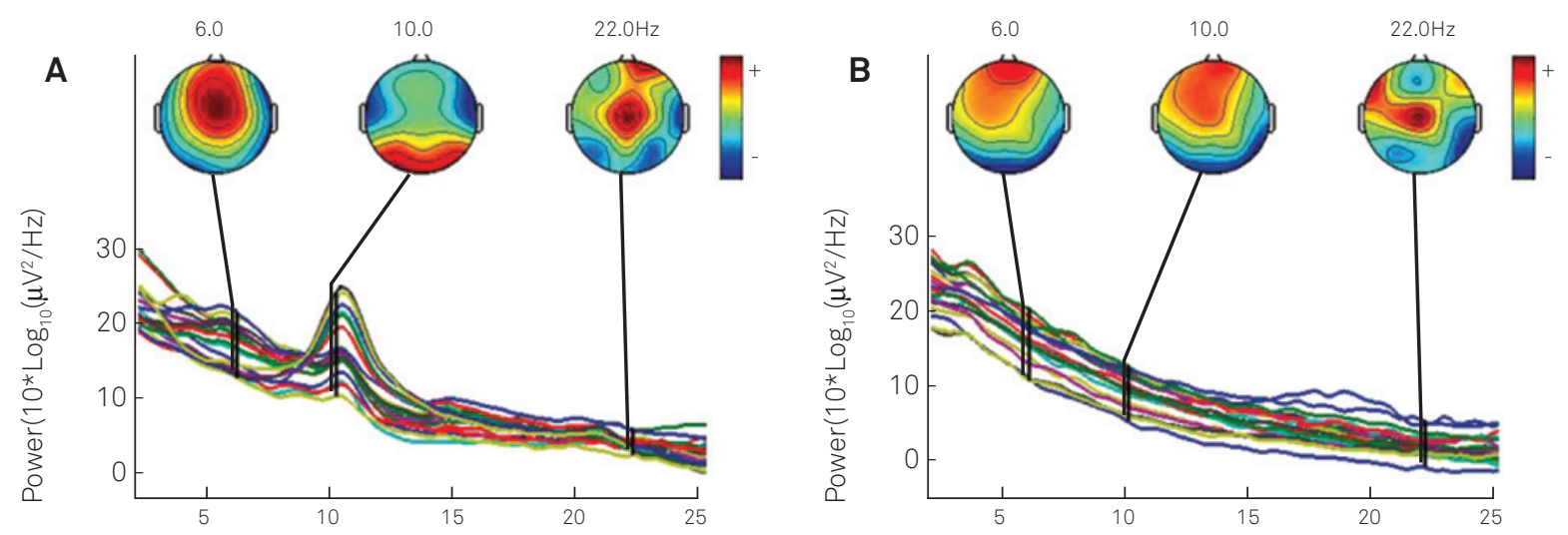

Figure 2. Examples of the power spectrum obtained from the EEG of patients with acute encephalopathy from Groups A and B. A. Patient of the Group A with favorable outcome (hospital discharge). Note the peak in the physiologic alpha frequency (10Hz). B. Patient of the Group B with unfavorable outcome (death). The main peak is in the delta frequency.

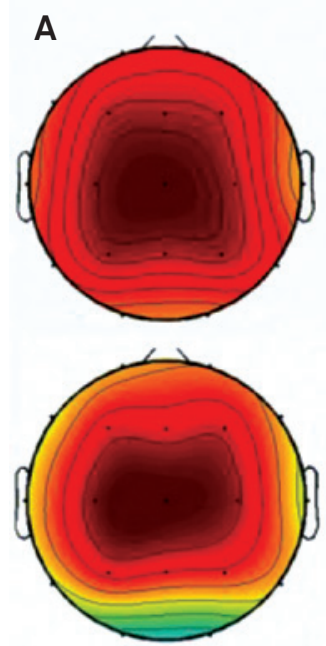

Group A

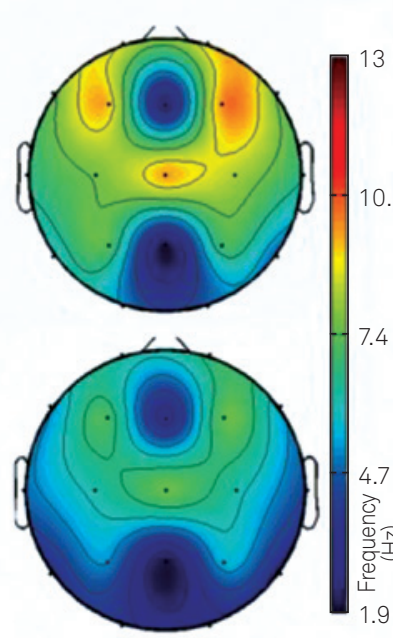

Group B
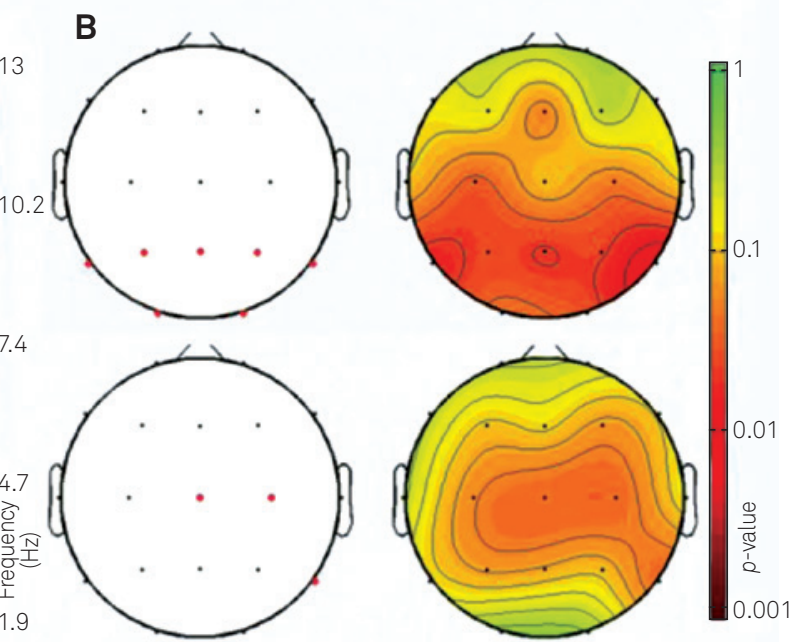

Statistical analysis

Figure 3. Results of the comparisons of the scalp maps / power spectrum between patients of Groups A and B. Superior row represents the comparisons between whole group A with group B. Inferior row illustrates the comparisons between patients of group A with systemic infectious diseases with group B. A) Results of the mean scalp maps showing predominance of fast activity in Group A as compared to Group B. The colored bar represents the frequencies depicted in the figure. B) Results of the statistical analysis. The red points on the left-hand model represent the electrodes that were statistically different when comparing the groups ( $p<0.05)$. The colored bar represents the $p$ value depicted at the right-hand model.

in $83 \%$ (5/6). Results of statistical comparisons with group B were similar to the whole group analysis. Spectral scalp maps of patients in this subgroup were more physiological and permutation analysis showed statistical differences in two central and one posterior electrode (Figure 3A and B - inferior row). Reduced power spectrum was also observed with the main differences occurring between 6 and 10Hz (Figure 4B).

\section{DISCUSSION}

The current investigation revealed two important findings. First, patients with AEs and poor outcome have distinct EEG patterns that are characterized by signs of severe encephalopathy. Although EEG has long been recognized as a useful prognostic tool, our study highlights that, in a general hospital setting with patients having pathologies of varied etiology, the EEG is a very useful complementary diagnostic tool. A second important result of the current work is a mapping of the quantitative abnormalities in two groups of AEs patients. Patients having a poor prognosis did not present with a physiological anterior posterior gradient on the EEG. In addition, we found an overall slowing of EEG frequencies in patients with a poor prognosis.

Given that the etiology of the AE may affect the EEG differently, a second comparison between patients with the same diagnosis in both groups was performed. The results were similar to the whole group analysis. The comparison of 

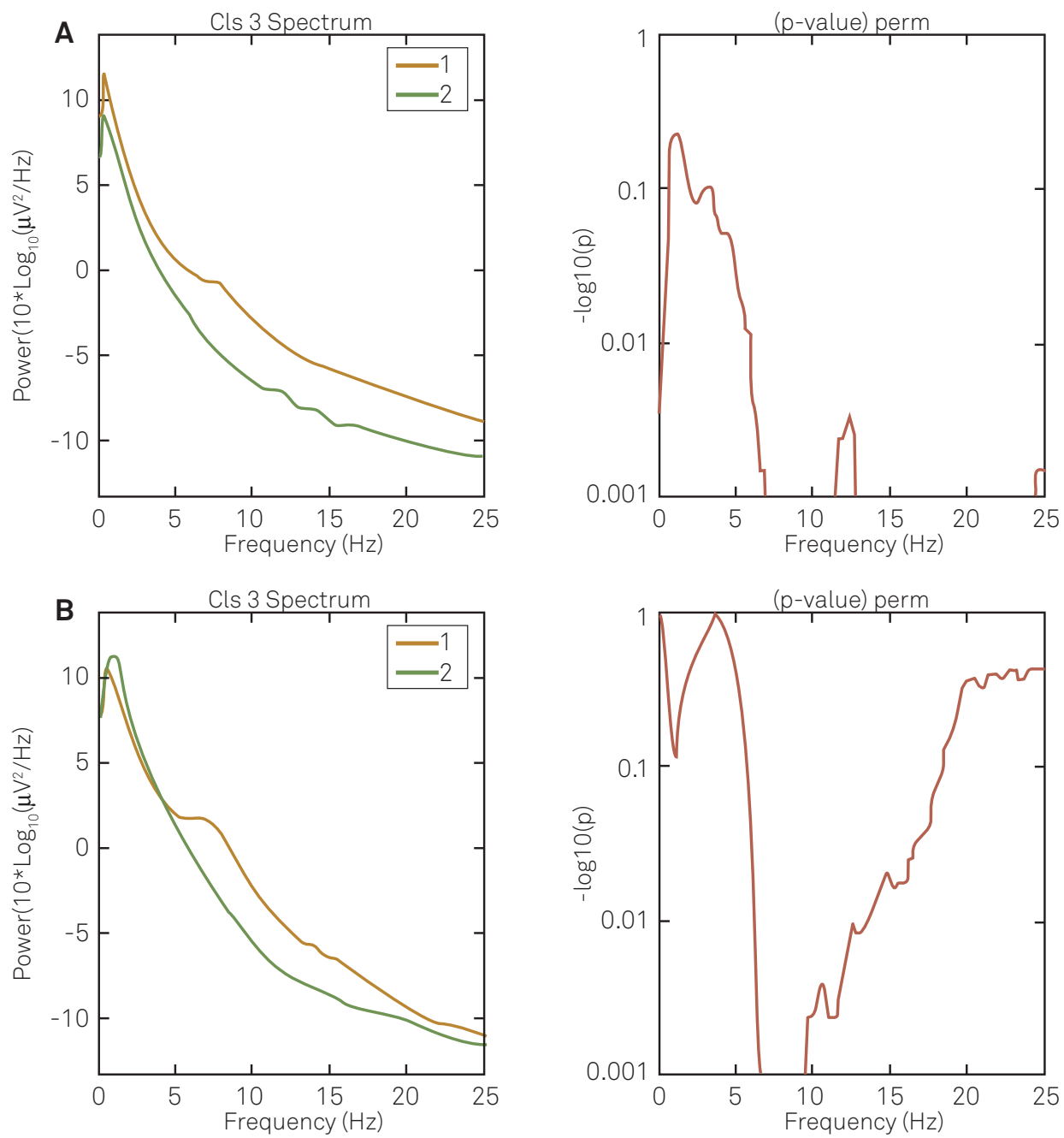

Figure 4. Comparison of the EEG power spectrum between Groups A (red line) and B (green line). The left figure represents the mean spectrum obtained from the main cluster of independent components. The right figure depicts the p values according to the EEG frequency. A) Comparisons between whole group A with group B. B) comparisons between patients of group A with systemic infectious diseases with group B.

the scalp maps of subgroup A (only patients with systemic infectious diseases) with group B showed differences only in two central and one posterior electrode(Figure 3B). However, the scalp maps are very similar showing a higher frequency in the group A in both analyses (Figure 3). Evaluation of the clustered independent components showed a better defined spectral peak in the subgroup A. Statistical analysis also showed main significance differences in the $6-10 \mathrm{~Hz}$ range (Figure 4). It is important to observe that qualitative analysis did not show differences between these groups.

Clinical evaluations of the AEs patients in the current study revealed a higher mortality in patients who had an elevated number of comorbidities. Interestingly, the average age of the patients did not differ between the groups. However, as the number of previous diseases naturally increases with age, mortality is most likely higher in older patients. The small number of patients in Group B likely contributed to this lack of difference. Different etiologies, as one would expect to find in a general hospital setting, contributed to the cause of the AEs in these patients. The etiologies reported in the two groups were similar to those observed in a previous investigation, with the exception of dementia and learning disabilities that were not observed in the current study ${ }^{14}$. Dementia and learning disabilities were the main underlying cause of the AEs observed amongst a previous investigation of 123 patients ${ }^{14}$.

One interesting finding from the current study is that all patients of Group B presented with toxic encephalopathies secondary to systemic infectious diseases. Evolution, or some degree of sepsis, contributed to this elevated mortality. Sepsis is often accompanied by an acute deterioration of mental status. The underlying mechanism of this is highly complex, and results from both inflammatory and non-inflammatory processes that induce blood-brain barrier breakdown, dysfunction of intracellular metabolism, neural cell death, and brain injuries. Sepsis-associated encephalopathy is accompanied by EEG abnormalities, and EEG exams are usually 
required in the presence of seizure and when encephalopathy remains unexplained ${ }^{15}$. Our results also suggest that the increasing use of qEEG in the context of sepsis related AEs could be useful in determining the severity and prognosis of this condition, as well as directing treatment goals. In addition, a previous investigation using this same group of patients demonstrated that the severity of EEG abnormalities has a clear relation with patient mortality ${ }^{16}$.

The current study has several drawbacks. One important limitation is that, the number of patients in group B was small. This difference may have introduced a bias in the statistical analysis. The groups also significantly differ in nonEEG parameters (comorbidities, etiology, and level of consciousness), and these likely are confounding factors with regard to outcome. Therefore, these limitations have to be considered when interpreting the results. Another important problem is that, while EEG has an excellent temporal resolution, it usually provides only a brief snapshot of brain activity, which may or may not be characteristic. Thus, a single recording is not the gold standard method for the evaluation of patients with abnormalities of consciousness, and an investigation using serial or continuous EEG monitoring would be more informative. Despite of these problems, in the current investigation, which was centered upon the detection of background abnormalities, the one-time recording paradigm was useful. Therefore, the value of a single EEG recording may not be diminished, especially when severe background abnormalities are present.

The objective of qEEG is to improve the detection of abnormalities in brain activity. In this study, the visual analysis of EEG traces showed similar results to the quantitative analysis. However, the main purpose of using qEEG in this investigation was to allow the comparison of EEGs between the two groups. Quantitative EEG has the advantage of not being user-dependent, thus the investigator bias is reduced. The quantitative analysis is informative because the whole EEG recording may be condensed into a single graphic, which is helpful for the interpretation of the exam by non-specialists. In addition, a key point of the qEEG is that differences between groups may be detected with more precision, as demonstrated in the current work.

Finally, the use of EEG must be routine in all patients with suspected AEs. This exam is useful not only for etiological diagnosis but also for the quantification of the severity of the AE. Older patients diagnosed with an AE with multiple comorbidities, particularly systemic infections, must be treated with intensive care, especially if the EEG shows signs of severe encephalopathy.

\section{References}

1. Guidotti M, Chiveri L, Mauri M. Acute encephalopathies. Neurol Sci 2006;27 (Suppl 1):S55-S56.

2. Posner JB, Plum F. Plum and Posner's diagnosis of stupor and coma. In: Posner JB, Plum F (Eds). Oxford ; New York: Oxford University Press, 2007.

3. Kaplan PW, Rossetti AO. EEG patterns and imaging correlations in encephalopathy: encephalopathy part II. J Clin Neurophysiol 2011;28:233-251.

4. Kaplan PW. The EEG in metabolic encephalopathy and coma. J Clin Neurophysiol 2004;21:307-318.

5. Brenner RP. Is it status? Epilepsia 2002;43(Suppl 3):S103-S113.

6. Brenner RP. The interpretation of the EEG in stupor and coma. Neurologist 2005;11:271-284.

7. Borges MA, Botos HJ, Bastos RF, Godoy MF, Marchi NS. Emergency EEG: study of survival. Arq Neuropsiquiatr 2010;68:174-178.

8. Niedermeyer E, Schomer DL, Lopes da Silva FH. Niedermeyer's electroencephalography : basic principles, clinical applications, and related fields. In: Niedermeyer E, Schomer DL, Lopes da Silva FH (Eds). Philadelphia: Wolters Kluwer/Lippincott Williams \& Wilkins, 2011.

9. Rossetti AO, Carrera E, Oddo M. Early EEG correlates of neuronal injury after brain anoxia. Neurology 2012;78:796-802.
10. Amodio P, Marchetti P, Del Piccolo F, et al. Spectral versus visual EEG analysis in mild hepatic encephalopathy. Clin Neurophysiol 1999;110:1334-1344.

11. Delorme A, Makeig S. EEGLAB: an open source toolbox for analysis of single-trial EEG dynamics including independent component analysis. J Neurosci Methods 2004;134:9-21.

12. Jung KY, KangJK, Kim JH, Im CH, Kim KH,Jung HK. Spatiotemporospectral characteristics of scalp ictal EEG in mesial temporal lobe epilepsy with hippocampal sclerosis. Brain Res 2009;1287:206-219.

13. Onton J, Westerfield M, Townsend J, Makeig S. Imaging human EEG dynamics using independent component analysis. Neurosci Biobehav Rev 2006;30:808-822.

14. O'Sullivan SS, Neligan A, Mullins GM, et al. Aetiology and prognosis of encephalopathic patterns on electroencephalogram in a general hospital. J Clin Neurosci 2008;15:637-642.

15. lacobone E, Bailly-Salin J, Polito A, Friedman D, Stevens RD, Sharshar T. Sepsis-associated encephalopathy and its differential diagnosis. Crit Care Med 2009;37(10 Suppl):S331-\$336.

16. Young GB, Bolton CF, Archibald YM, Austin TW, Wells GA. The electroencephalogram in sepsis-associated encephalopathy. J Clin Neurophysiol 1992;9:145-152. 